\title{
Image Compression with Haar Wavelet Transform
}

\author{
Y. Shantikumar Singh \\ Department of ECE, \\ NIT Manipur, India
}

\author{
B. Pushpa Devi \\ Department of ECE \\ NIT Meghalya, India
}

\author{
Kh. Manglem Singh \\ Department of CSE \\ NIT Manipur, India
}

\begin{abstract}
Wavelet transform is a mathematical tool for hierarchically decomposing functions. Wavelet transform has been proved to be a very useful tool for image processing in recent years. The most distinctive feature of Haar Transform lies in the fact that it lends itself easily to simple manual calculations. The aim of this paper is to describe the algorithm for image compression using Haar Transform. The quality analysis of this method has been checked for three different levels of HT scaling with varying quantization with threshold encoding scheme. In this paper, the image quality analysis is done using two sets of parameters, namely the popular peak signal to noise ratio and compression ratio.
\end{abstract}

\section{Keywords}

Haar Wavelet Transform, Compression ratio, PSNR and MSE.

\section{INTRODUCTION}

Image compression is a process of efficiently coding digital images [1] to reduce the number of bits required [2] in representing an image. Image compression plays a vital role in several important and diverse applications, including televideoconferencing, remote sensing, medical imaging $[1$, 4] and magnetic resonance imaging [5]. With the increase of spatial resolution and swath, the space missions are faced with the necessity of handling an extensive amount of imaging data. A digital image obtained by sampling and quantizing a continuous tone picture requires an enormous storage. For instance, a 24 bit colour image with 512 x 512 pixels will occupy $768 \mathrm{~KB}$ storage on a disk and without compression only 911 such pictures can fit in a single compact disc. To transmit such an image over a $28.8 \mathrm{Kbps}$, a modem would take almost 4 minutes. The wavelet Transform, developed for signal and image processing, has been extended for use on relational data sets $[6,7]$. Image compression research aims at reducing the number of bits needed to represent an image by removing the spatial and spectral redundancies as much as possible. The basic idea behind the image compression is that in most of the images we find that their neighboring pixels are highly correlated and have redundant information [8].

\section{HAAR WAVELET TRANSFORM}

The Haar Wavelet Transform (HT) is one of the simplest and basic transformations from the space domain to a local frequency domain. The most distinctive feature of Haar Transform lies in the fact that it lends itself easily to simple manual calculation. Since the Haar Transform is memory sufficient, exactly reversible without the edge effects, it is fast and simple. As such the Haar Transform technique is widely used these days in wavelet analysis. A HT decomposes each signal into two components; one is called average (approximation) or fluctuation. A precise formula for the values of first average sub signal, $a^{l}=\left(a_{1}, a_{2}, \ldots, a_{N / 2}\right)$, at one level for a signal of length $N$ i.e. $f=\left(f_{1}, f_{2}, \ldots, f_{N}\right)$ is

$$
a_{n}=\frac{f_{2 n-1+f_{2 n} n}}{\sqrt{2}}, n=1,2,3, \ldots, N / 2,
$$

and the first detail sub signal, $\mathrm{d}^{1}=\left(\mathrm{d}_{1}, \mathrm{~d}_{2}, \ldots, \mathrm{d}_{\mathrm{N} / 2}\right)$, at the same level is given as

$$
d_{n}=\frac{f_{2 n-1-f_{2 n}}}{\sqrt{2}}, \mathrm{n}=1,2,3, \ldots, N / 2 .
$$

\subsection{Steps for Haar Wavelet Transform}

Step 1 . Find the average of each pair of samples. ( $n / 2$ averages)

Step 2. Find the difference between each average and samples which was calculated from ( $n / 2$ differences).

Step 3. Fill the first half of the array with averages.

Step 4. Fill the first half of the array with differences.

Step 5. Repeat the process on the first half of the array. (The array length should be a power of two)

\subsection{Haar Wavelet Function}

The family of $N$ Haar functions $h_{k}(t)$ are defined on the interval $0 \leq t \leq 1$. The shape of the Haar function, of an index $k$, is determined by two parameters: $p$ and $q$,

where

$$
k=2^{p}+q-1
$$

and $k$ is in a range of $k=0,1,2, \cdots, N-1$.

When $k=0$, the Haar function is defined as a constant $h_{0}(t)=1 / \sqrt{N}$; when $\mathrm{k}>0$, the Haar function is defined as

$$
h_{k}(t)=\frac{1}{\sqrt{N}}\left\{\begin{array}{cl}
2^{p / 2} & (q-1) / 2^{p} \leq t<(q-0.5) / 2^{p} \\
-2^{p / 2} & (q-0.5) / 2^{p} \leq t<q / 2^{p} \\
0 & \text { otherwise }
\end{array}\right.
$$

From the above equation, one can see that $p$ determines the amplitude and width of the non-zero part of the function, while $q$ determines the position of the non-zero part of the Haar function.

\subsection{The Haar Transform Matrix}

The discrete Haar functions formed the basis of the Haar matrix $H$

$$
\begin{aligned}
H_{2 N} & =\left[\begin{array}{c}
\mathrm{H}_{\mathrm{N}} \otimes[1,1] \\
\mathrm{I}_{\mathrm{N}} \otimes[1,-1]
\end{array}\right] \\
H(0) & =1
\end{aligned}
$$


where

$$
I_{N}=\left[\begin{array}{ccccc}
1 & 0 & \cdots & 0 & 0 \\
0 & 1 & \cdots & 0 & 0 \\
\vdots & \vdots & \ddots & \vdots & \vdots \\
0 & 0 & \cdots & 1 & 0 \\
0 & 0 & \cdots & 0 & 1
\end{array}\right]
$$

And $\otimes$ is the Kronecker product.

The Kronecker product of $A \otimes B$, where $A$ is an $\mathrm{m} \times \mathrm{n}$ matrix and $B$ is a $\mathrm{p} \times \mathrm{q}$ matrix, is expressed as

$$
A \otimes B=\left[\begin{array}{ccc}
a_{11} B & \cdots & a_{1 n} B \\
\vdots & \ddots & \vdots \\
a_{m 1} B & \cdots & a_{m n} B
\end{array}\right]
$$

When $N=2^{k}$

$$
H_{N}=\left[\begin{array}{c}
\phi \\
\mathrm{h}_{0,0} \\
\mathrm{~h}_{1,0} \\
\mathrm{~h}_{1,1} \\
\vdots \\
\mathrm{h}_{\mathrm{k}-1,0} \\
\mathrm{~h}_{\mathrm{k}-1,1} \\
\vdots \\
\mathrm{h}_{\mathrm{k}-1,2^{\mathrm{k}-1}-1}
\end{array}\right]
$$

where $\phi=\left[\begin{array}{lllll}1 & 1 & 1 & \cdots & 1\end{array}\right]$ is a $1 \times N$ matrix, and $h_{p, q}[n]$ is a Haar function.

The Haar matrix is real and orthogonal, i.e.

$$
\begin{gathered}
H=H^{*} \\
H^{-1}=H^{T} \text { i.e. } H^{T} H=I
\end{gathered}
$$

An un-normalized 8-point Haar matrix $H_{8}$ is

$$
H[m, n]=\left[\begin{array}{cccccccc}
1 & 1 & 1 & 1 & 1 & 1 & 1 & 1 \\
1 & 1 & 1 & 1 & -1 & -1 & -1 & -1 \\
1 & 1 & -1 & -1 & 0 & 0 & 0 & 0 \\
0 & 0 & 0 & 0 & 1 & 1 & -1 & -1 \\
1 & -1 & 0 & 0 & 0 & 0 & 0 & 0 \\
0 & 0 & 1 & -1 & 0 & 0 & 0 & 0 \\
0 & 0 & 0 & 0 & 1 & -1 & 0 & 0 \\
0 & 0 & 0 & 0 & 0 & 0 & 1 & -1
\end{array}\right]
$$

From the definition of the Haar matrix $H$, one can observe that, unlike the Fourier transform, $H$ matrix has only real element (i.e., 1, -1 or 0 ) and is non-symmetric.

The first row of $H$ matrix measures the average value, and the second row $H$ matrix measures a low frequency component of the input vector. The next two rows are sensitive to the first and second half of the input vector respectively, which corresponds to moderate frequency components. The remaining four rows are sensitive to the four section of the input vector, which corresponds to high frequency components.

\subsection{Image Quality Measurement}

Compression ratio is used to quantify the reduction in data representation size. Throughout this paper, numeric values are given for two measures of compression performance compression ratio (CR) and peak signal to noise ratio (PSNR). If $\mathrm{N}_{1}$ and $\mathrm{N}_{2}$ denote the number of information carrying units in original and compressed image respectively, then the compression ratio can be defined by (1)

$$
\text { Compression Ratio }=\frac{N_{1}}{N_{2}}
$$

Mean square error is a criterion for an estimator: the choice is the one that minimizes the sum of squared errors due to bias and due to variance. As a loss function, MSE is called squared error loss. MSE measures the average of the square of the "error. The MSE is the second moment (about the origin) of the error, and thus incorporates both the variance of the estimator and its bias. For an unbiased estimator, the MSE is the variance. In an analogy to standard deviation, taking the square root of MSE yields the root mean squared error or RMSE. This has the same units as the quantity being estimated. For an unbiased estimator, the RMSE is the square root of the variance, known as the standard error.

$M S E=\frac{1}{m n} \sum_{i=0}^{m-1} \sum_{j=0}^{n-1}\|I(i, j)-R(i, j)\|^{2}$

Where $\mathrm{m} \mathrm{x} \mathrm{n}$ is the image size and $\mathrm{I}(\mathrm{i}, \mathrm{j})$ is the input image and $R(i, j)$ is the retrieved image.

Here, we define the Peak signal-to-noise ratio (PSNR) in the near-lossless compression, the measure of image quality, as

$$
\text { PSNR }=20 * \log _{10}\left\{\frac{255}{\sum_{0}^{m} \sum_{0}^{n}(I-R)^{2}}\right\}
$$

Where, I and $\mathrm{R}$ are the original and reconstructed images with a height of $m$ and width of $n$ respectively. As the PSNR value increases the reconstructed image quality increases.

\section{RESULT AND DISCUSSION}

Compression ratio (CR), Mean Square Error (MSE) ,Peak Signal-to-noise Ratio (PSNR) and Bit per Pixel (BPP) are computed after certain number of multiple-level decomposition for a number of images using equation (1), (2),(3) and (4) respectively.

For evaluation standard test image Lena is used.

Table 1. Performance and Compression of some test images.

\begin{tabular}{|l|c|l|l|l|l|l|}
\hline \multicolumn{1}{|c|}{ Image } & Level & PERF1 & PERF2 & PSNR & MSE & CR \\
\hline lena.png & 1 & 20.643 & 99.999 & 44.312 & 2.409 & 6.348 \\
\hline Akiyo.jpg & 1 & 59.027 & 99.999 & 48.289 & 0.964 & 3.277 \\
\hline lena.png & 2 & 22.847 & 99.999 & 49.743 & 0.689 & 6.172 \\
\hline Akiyo.jpg & 2 & 68.438 & 99.999 & 51.490 & 0.461 & 2.524 \\
\hline lena.png & 3 & 23.193 & 99.999 & 52.630 & 0.177 & 6.144 \\
\hline Akiyo.jpg & 3 & 69.519 & 99.997 & 56.343 & 0.150 & 2.438 \\
\hline lena.png & 4 & 36.668 & 99.994 & 55.618 & 0.178 & 5.066 \\
\hline Akiyo.jpg & 4 & 69.622 & 99.999 & 61.742 & 0.043 & 2.430 \\
\hline
\end{tabular}




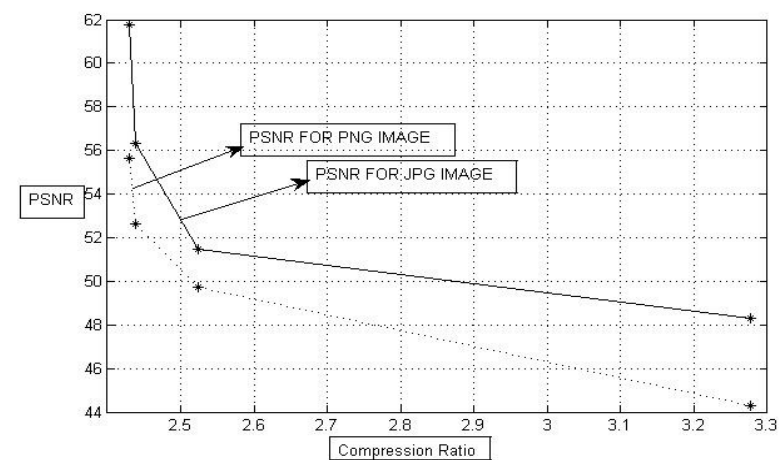

Figure 1: Performance of compression ratio for lena.png and Akiyo.jpg images.

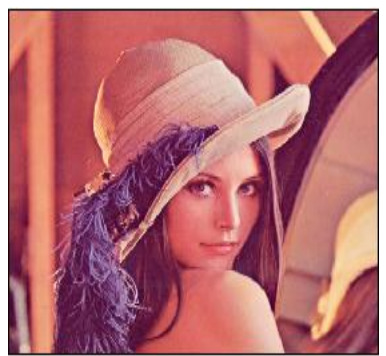

Original Image

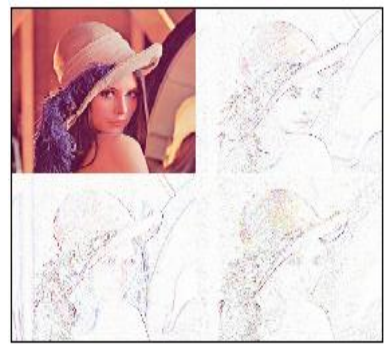

Original decomposition Image

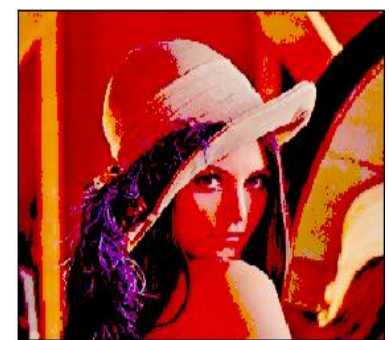

Compressed Image

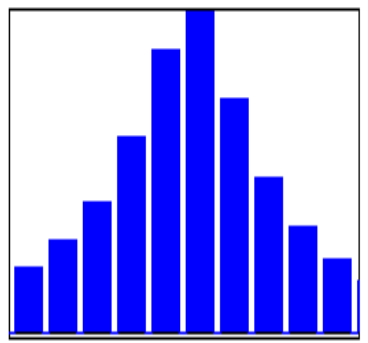

Normalized Histogram

Figure 2: Compression image for decomposition level 1

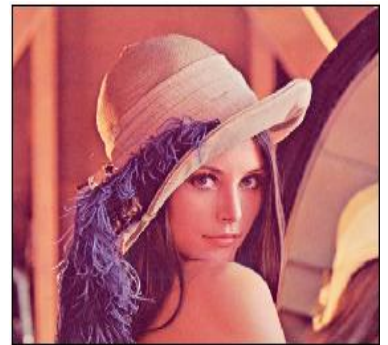

Original Image

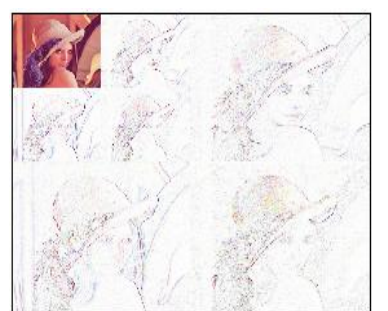

Original decomposition Image

Figure 3: Compression image for decomposition level 2

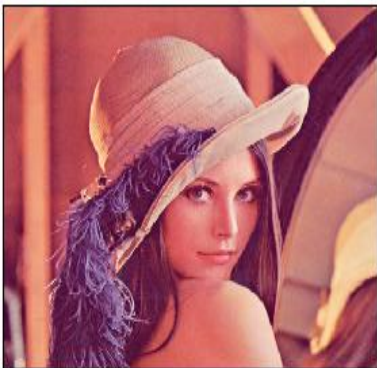

Original Image

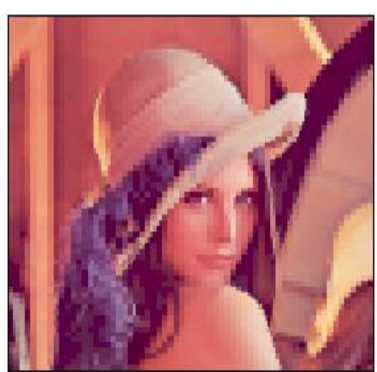

Compressed Image

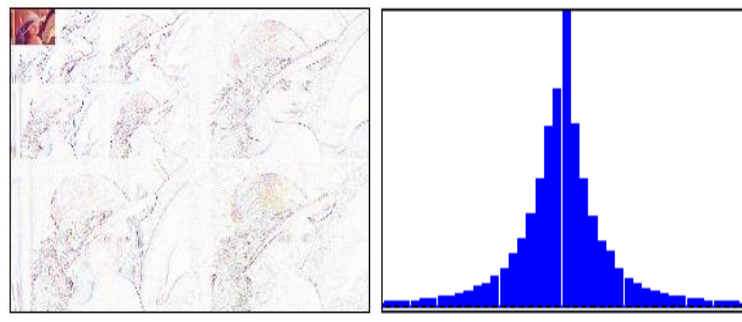

Original decomposition Image Normalized Histogram

Figure 4: Compression image for decomposition level 3

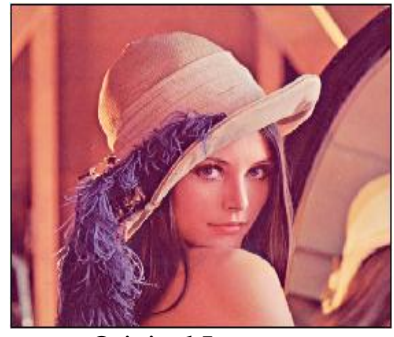

Original Image

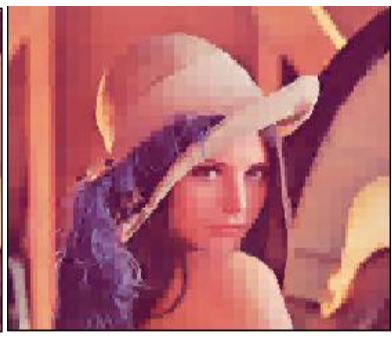

Compressed Image

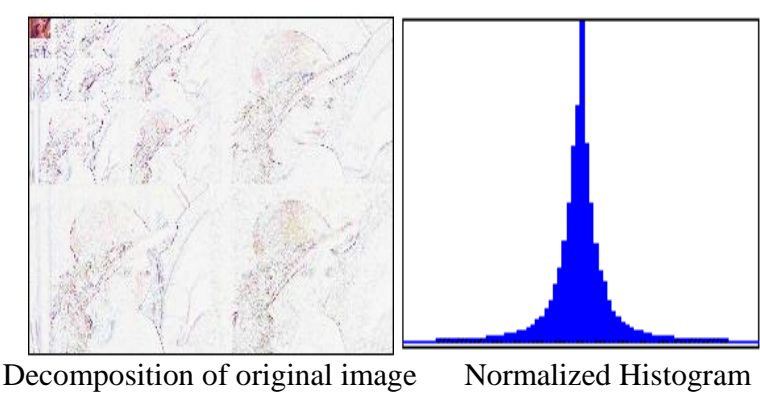

Figure 5: Compression image for decomposition level 4

\section{CONCLUSION}

In this paper, we have presented the basic and applied research in the field of wavelet has made tremendous progress in the last decade. Image compression schemes based on wavelets are rapidly gaining maturity and have already began to appear in commercial software/hardware systems. The reconstruction quality of wavelet images has become better than jpeg which is the current international standard for image compression. In this paper multiple-level decomposition of images and compression of images using Haar wavelet Transform has been shown.

By considering several input images, it is observed that MSE is low and PSNR is high in Haar Wavelet Transform based compression. This paper also presents the simplicity of image compression with HT. From the simulation result we 
conclude that HT is a mathematical tool for image decomposition. The results are found that Haar wavelet Transform has better performance in image compression than known standards for image compression.

\section{ACKNOWLEDGEMENT}

The author thanks L. Surajkumar Singh, Assistant Professor of ECE department, NIT Manipur for his guidance and active support during the progress of our research. Without his support and encouragement this research would have been trivial. The author also thanks Dr. Y. Rohen Singh, HOD of Mathematics department, NIT Manipur for his cooperation and guidance. We would also like to mention that it would not have been possible without the timely help and support of Electronics and Communication Engineering department Labs, especially the Computer Lab.

\section{REFERENCES}

[1] M.G.L.Grewe and J.P. Thiran, 2000. Multirate Coding of 3D Medical Data. In proceedings of the 2000 International Conference on Image Processing, IEEE, 3:656-659.

[2] A.Bhardwaj and R. Ali, Image Compression Using Modified Fast Haar Wavelet Transform, World Applied Science Journal 7 (5): 647-653, 2009 ISSN 1818-4952.

[3] K. Lees "Digital compression using Wavelets', Report of M.S.2002.

[4] Wang, J. and H.K. Huang, 1996. Medical Image Compression by using 3 Dimension Wavelet Transform. IEEE Transactions on Medical Imaging, 15 (4): 547-554.

[5] B.E.J. Fadili, V.Maxim, L.Sendur, J. Sucking, B. Whitcher, M. Brammer and M. Breakspear, 2004. Wavelets and Functional NeuroImage, 23 (1): 234-249.

[6] Joe.M.J., K.Y. Whang and S.W. Kim, 2001. Wavelet Transformation Based Management of Integrated Summary Data for Distributed Query Processing. Data and Knowledge Engg. 39 (3): 293-312.

[7] Vitter, J.S. and M. Wang, 1999. Approximate Computation of Multidimensional Aggregates of Sparse Data using Wavelets. In proceedings of the ACM
SIGMOD International Conference on Management of Data, pp: 193-204.

[8] Saha S., 2000. Image Compression from DCT to Wavelets:A Review. ACM Cross Words Students Magazine, 6 (3).

[9] Vandendrope, L., b. Maison and F. Labeau, 1997. An Adaptive Transform Approach for Image Compression. IEEE Digital Signal Processing Workshop, pp: 41-44.

[10] S. Mallat, in: A Wavelet Tour of Signal Processing, Academic Press, UK, 1999.

[11] W.B. Pennebaker, J.L. Mitchell, in: JPEG: Still Image Data Compression Standard, Van Nostrand Reinhold, USA, 1993.

[12] D.S. Taubman, M.W. Marcellin, in: JPEG2000 Image Compression Funda-mentals, Standards and Practice, Kluwer Academic Publishers, USA, 2002.

[13] K.C. Liang, C.-C.J. Kuo, WaveGuide: a joint waveletbased image representa-tion and description system, IEEE Transactions on Image Processing 8 (11) (1999) $1619-1629$.

[14] Kaiser, G., 1998. The Fast Haar Transform: Gateway to Wavelet. Potentials, IEEE, 17 (2): 34-37.

[15] Galli, A.W., G.T. Heydt and P.F. Ribeiro, 1996 Exploring the Power of Wavelet Analysis. IEEE Computer Application in Power, pp: 37-41.

[16] Benedetto, John J. and Frazier, Michael (editors), Wavelets; Mathematics and Applications, CRC Press, Boca RatonFL, 1996.

[17] Rafael C. Gonzalez, Richard E. Woods. (1992), Digital Image processing ( $2^{\text {nd }}$ edition), NJ: Prentice Hall.

[18] Locker Gnome (2011), "Real World Application of Image Compression".

[19] Lawson S and Zhu J (2002) Image compression using wavelets and JPEG2000: a tutorial. Electrn.Commun. Engg. J. 5(1) 113-123. 